03

\title{
Устойчивость оптимального аэродинамического проектирования изолированного трехмерного крыла к его начальной форме
}

\author{
(C) С.В. Пейгин, С.В. Тимченко, Б.С. Эпштейн \\ ООО „ОПТИМЕНГА-777“, \\ 143026 Москва, Россия \\ e-mail: mishpahat_peiguine@yahoo.com
}

(Поступило в Редакцию 4 апреля 2018 г.)

\begin{abstract}
Приведены результаты исследования устойчивости алгоритма оптимального аэродинамического проектирования изолированного трехмерного крыла широкофюзеляжного дальнемагистрального самолета к его начальной форме. Решение задачи определения геометрии, доставляющей минимум полного сопротивления крыла при фиксированном коэффициенте подъемной силы с учетом многочисленных аэродинамических и геометрических ограничений, было получено с помощью алгоритма, сочетающего в себе методы высокоточного математического моделирования и глобального оптимального поиска с использованием суперкомпьютерных технологий. Было показано, что алгоритм устойчив к выбору начальной формы крыла, поскольку оптимальные геометрии, полученные для двух сильно отличающихся вариантов задания начальной формы крыла, очень близки друг к другу и обладают практически идентичными интегральными аэродинамическими характеристиками как в основной точке проектирования, так и в широком диапазоне условий полета.
\end{abstract}

DOI: 10.21883/JTF.2018.12.46779.136-18

\section{Введение}

Процесс проектирования летательного аппарата, делится на три последовательных этапа [1]: концептуального (эскизного) проектирования (на котором принимается решение о характере компоновки и о форме крыла в плане), предварительного проектирования (на котором в ходе аэродинамического проектирования определяется форма планера летательного аппарата) и финального детального проектирования. Решающим среди них является второй этап, по окончании которого дается окончательный ответ на вопрос: удалось ли найти форму, которая гарантированно удовлетворяет всем требованиям к самолету по дальности, грузоподъемности и энергоэффективности или нет. Фактически, по завершении этого принимается окончательное решение о судьбе проекта. Поэтому понятно, что успешное завершение стадии предварительного проектирования и достижение всех целей, поставленных перед этой стадией проекта, является ключевым фактором для успеха проекта создания летательного аппарата в целом.

Математически задача аэродинамического проектирования заключается в оптимизации формы летательного аппарата по полному сопротивлению с учетом конструктивных параметров и конструктивных ограничений. Первостепенная важность снижения аэродинамического сопротивления самолета становится ясной из так называемой формулы Бреге [1]: при сохранении дальности полета, уменьшение сопротивления самолета на 1\% ведет к увеличению его полезной нагрузки на 7.6\%.

В силу актуальности и практической востребованности, разработка методов оптимального аэродинамического проектирования ведется рядом исследовательских коллективов в мире. Среди них можно указать группу проф. А. Джеймсона (A. Jameson) из Станфордского университета в США [1,2], группу проф. В. Mohammadi и проф. О. Pironneau из Франции $[3,4]$ и группу доктора N. Kroll из DLR (German Aerospace Center) [5,6]. Анализ этих работ показывает, что решения этих групп по большей части основаны на детерминистическом подходе к оптимизации с использованием градиентного метода при поиске оптимума; эти решения являются локальными и в силу этого не обеспечивают нахождение глобального оптимума; степень зрелости этих решений не позволяет применить их для решения реальных задач.

Исходя из этого, в целом можно сделать вывод, что метод оптимального аэродинамического моделирования, используемый в настоящей работе не имеют аналогов в мире, поскольку расчеты аэродинамических характеристик в процессе проектирования основаны на численном решении осредненных по Рейнольдсу уравнений Навье-Стокса с использованием конечноразностной схемы повышенного порядка точности; нахождение оптимальной формы базируется на глобальном методе поиска с учетом произвольного числа аэродинамических и геометрических ограничений; подход обладает высокой вычислительной эффективностью, позволяющей получать решение в сжатые сроки; метод применим к широкому диапазону изменения проектируемых конфигураций по геометрической сложности.

\section{Математическая постановка задачи}

Каждый цикл аэродинамического проектирования стартует с начальной компьютерной геометрической модели летательного аппарата. В первом таком цикле 
геометрическая модель поступает из стадии концептуального дизайна вместе с аэродинамическими характеристиками дизайна. В число аэродинамических характеристик входят заданные значения коэффициента подъемной силы и числа Маха набегающего потока, а также высота полета и максимально допустимое значение сопротивления в крейсерском режиме полета. Эти данные призваны обеспечить выполнение аэродинамических целей полета (таких как дальность, полезная нагрузка, объем топливного бака и т.д.). Поиск искомой геометрической формы осуществляется в классе решений, удовлетворяющим различным геометрическим, аэродинамическим и междисциплинарным ограничениям, которые также определяются на стадии концептуального дизайна. Обычно ограничения накладываются на толщину профилей, допустимые значения момента тангажа и коэффициента подъемной силы при взлете и т.д.

Сформулируем в точных терминах проблему оптимизации.

Начнем с одноточечной задачи. Ее цель - найти аэродинамическую форму, которая минимизирует коэффициент полного сопротивления $C_{x}$ с учетом следующих аэродинамических и геометрических ограничений:

- аэродинамические ограничения: заданный постоянный коэффициент подъемной силы $C_{y}$ и максимально допустимый момент тангажа $M_{Z}$;

- геометрические ограничения на следующие величины задаваемые для каждой - оптимизируемой секции крыла:

- относительная толщина $(t / c)_{i}$,

- радиус кривизны передней кромки $\left(R_{L}\right)_{i}$,

- угол задней кромки $\left(q_{T}\right)_{i}$,

- локальные толщины $(y / t)_{i j}$.

В этих ограничениях $i=1, \ldots, N_{w s}$ - число секций по размаху крыла, а $j=1, \ldots, N_{b s}(i)$ - число ограничений на локальную толщину в секции номер $i$.

Цель многоточечной оптимизации - минимизировать взвешенную комбинацию коэффициентов сопротивления в нескольких точках дизайна. При этом геометрические ограничения не зависят от точки дизайна, а аэродинамические ограничения задаются для каждой точки дизайна по отдельности.

Отметим, что решение этой задачи для реальных конфигураций является сложным и весьма нетривиальным, поскольку точный расчет сопротивления очень сложен для реальных конфигураций; нет общего решения проблемы глобального геометрического представления аэродинамических поверхностей; оптимальный поиск происходит в пространстве высокой размерности; необходим эффективный учет большого числа нелинейных ограничений; решение задачи требует огромнейшего объема вычислений.

В настоящей работе использовался алгоритм оптимального аэродинамического проектирования, который состоял из трех основных элементов:

- вычисление аэродинамических характеристик тестируемой геометрии с использованием численного реше- ния осредненных по числу Рейнольдса полных уравнений Навье-Стокса;

- поиск оптимальной геометрии на базе генетического алгоритма с учетом заданных многочисленных ограничений различного типа на оптимальное решение;

- многоуровневой параллелизации вычислительного потока на многопроцессорном вычислительном кластеpe.

Выбор адекватной математической модели, описывающей течение около летательного аппарата, также является компромиссом между необходимой точностью описания и сложностью решения соответствующей начальнокраевой задачи. В нашем случае, поскольку для расчета целевой функции $C_{x}$ необходимо учитывать эффекты вязко-невязкого взаимодействия, было принято решение использовать в качестве базовых уравнений осредненные по числу Рейнольдса уравнения Навье-Стокса.

Уравнения Навье-Стокса вязкой сжимаемой жидкости могут быть записаны в следующей форме:

$$
\mathbf{q}_{t}+\operatorname{div} \mathbf{C}=\operatorname{div} \mathbf{V}
$$

где тензор $\mathbf{C}=(\mathbf{f}, \mathbf{g}, \mathbf{h})$ содержит конвективные члены, тензор $\mathbf{V}=(\mathbf{r}, \mathbf{s}, \mathbf{t})$ содержит вязкие члены, $\mathbf{q}=(\rho, \rho u, \rho v, \rho w, E)^{T}, \rho-$ плотность,$(u, v, w)-$ вектор скорости, $E$ - энергия, $t-$ время, $\mathbf{f}, \mathbf{g}, \mathbf{h}-$ невязкие (конвективные) потоки, $\mathbf{r}, \mathbf{s}, \mathbf{t}-$ вязкие потоки.

Компоненты невязких потоков имеют вид

$$
\begin{aligned}
\mathbf{f}(\mathbf{q}) & =u \mathbf{q}+p(0,1,0,0, u)^{T}, \\
\mathbf{g}(\mathbf{q}) & =v \mathbf{q}+p(0,0,1,0, v)^{T}, \\
\mathbf{h}(\mathbf{q}) & =w \mathbf{q}+p(0,0,0,1, w)^{T}
\end{aligned}
$$

Компоненты вязких потоков имеют вид

$$
\begin{aligned}
& \mathbf{r}(\mathbf{q})=\mu\left(0, \tau_{11}, \tau_{21}, \tau_{31}, \mu_{1}\right)^{T}, \\
& \mathbf{s}(\mathbf{q})=\mu\left(0, \tau_{12}, \tau_{22}, \tau_{32}, \mu_{2}\right)^{T}, \\
& \mathbf{t}(\mathbf{q})=\mu\left(0, \tau_{13}, \tau_{23}, \tau_{33}, \mu_{3}\right)^{T},
\end{aligned}
$$

где составляющие тензора вязких напряжений задаются следующим образом:

$$
\begin{gathered}
\tau_{11}=(4 / 3) u_{x}-(2 / 3) v_{y}-(2 / 3) w_{z}, \\
\tau_{21}=\tau_{12}=u_{y}+v_{x}, \\
\tau_{31}=\tau_{13}=u_{z}+w_{x}, \\
\tau_{22}=(4 / 3) v_{y}-(2 / 3) u_{x}-(2 / 3) w_{z}, \\
\tau_{32}=\tau_{23}=u_{z}+w_{y}, \\
\tau_{33}=(4 / 3) w_{z}-(2 / 3) u_{x}-(2 / 3) v_{y}, \\
\mu_{2}=u \tau_{11}+v \tau_{12}+w \tau_{13}+(c 2)_{x} /((\gamma-1) \operatorname{Pr}), \\
\mu_{3}=u \tau_{31}+v \tau_{32}+w \tau_{23}+(c 2)_{y} /((\gamma-1) P r),
\end{gathered}
$$


В этих формулах $\mu-$ значение вязкости, $\gamma-$ отношение теплоемкостей газа, $\operatorname{Pr}$ - число Прандтля,

$$
\begin{gathered}
p=(\gamma-1)\left[E-0.5 \rho\left(u^{2}+v^{2}+w^{2}\right],\right. \\
c^{2}=\gamma p / \rho, \\
H=(E+p) / \rho .
\end{gathered}
$$

Как уже отмечалось, точный расчет сопротивления реальных конфигураций до сих пор является непростой задачей. Причина этого довольно тривиальна. В отличие от остальных интегральных аэродинамических характеристик (таких как коэффициент подъемной силы, момент тангажа и т.д.), имеющих величину порядка $O(1)$, коэффициент полного аэродинамического сопротивления имеет величину на 2 порядка меньше (в районе 0.02). Поэтому, если не предпринимать специальных и весьма нетривиальных мер, численный метод, обеспечивающий точность 2 знака после запятой, не в состоянии обеспечить расчет сопротивления с необходимой для практики точностью $10^{-4}-10^{-5}$.

Поэтому правильный выбор численного метода решения осредненных по числу Рейнольдса полных уравнений Навье-Стокса вязкой сжимаемой жидкости в турбулентном режиме является одним из ключевых факторов успешного решения всей задачи оптимального проектирования.

В связи с этим в основе выбранного нами надежного, высокоточного и вычислительно-эффективного численного метода лежала схема высокого порядка точности ENO (Essentially Non-Oscilatory Scheme) в сочетании с многосеточным подходом (multigrid approach) с использованием многоблочных структурированных вычислительных сеток.

Основными характеристиками метода являются использование многоблочных структурированных сеток, многосеточного (многоуровневого) подхода, схемы высокого порядка точности, учет турбулентности и глубокая параллелизация.

В алгоритме кода конвективная часть уравнений аппроксимируется характеристическим оператором первого порядка, который легко обращается. Эта схема применяется везде при многосеточной релаксации. При этом схема высокого порядка ENO/WENO определяет поправки в правую часть дискретного уравнения только на самом тонком сеточном уровне, а вязкие члены аппроксимируются обычным образом.

Код обеспечивает точный расчет сопротивления для сложных аэродинамических конфигураций, достигая хорошей точности на грубых сетках. В нем отсутствуют искусственные параметры. Все эти качества делают код подходящим для индустриальных приложений. Данный код был успешно верифицирован в рамках AIAA Drag Prediction Workshop (своеобразного чемпионата мира среди кодов для расчета сопротивления реальных самолетных конфигураций). Проведенные сравнения с кодом OVERFLOW (сертифицированным кодом компании Boeing) дополнительно подтвердили точность этого кода [7].

При дискретизации сжимаемых уравнений Навье-Стокса для сжимаемого газа используется метод конечных объемов с явной схемой аппроксимации потоков.

Рассмотрим структурированную расчетную сетку, состоящую из ячеек, которые образуют структуру типа $(i, j, k)$.

Интегрируя по каждой ячейке, мы получаем систему обыкновенных дифференциальных уравнений, к которой применяется процедура интегрирования по времени.

Запишем аппроксимацию для ячейки с индексами $i, j, k$ :

$$
\begin{aligned}
& \left(\Omega_{i, j, k} \mathbf{q}_{i, j, k}\right)_{t}+[\mathbf{C} \cdot(S \mathbf{n})]_{i+0.5, j, k}-[\mathbf{C} \cdot(S \mathbf{n})]_{i-0.5, j, k} \\
& +\left[\mathbf{C} \cdot(S \mathbf{n})_{i, j+0: 5, k}-[\mathbf{C} \cdot(S \mathbf{n})]_{i, j-0.5, k}+[\mathbf{C} \cdot(S \mathbf{n})]_{i, j, k+0.5}\right. \\
& -[\mathbf{C} \cdot(S \mathbf{n})]_{i, j, k-0.5}=[\mathbf{V} \cdot(S \mathbf{n})]_{i+0.5, j, k}-[\mathbf{V} \cdot(S \mathbf{n})]_{i-0.5, j, k} \\
& +[\mathbf{V} \cdot(S \mathbf{n})]_{i, j+0: 5, k}-[\mathbf{V} \cdot(S \mathbf{n})]_{i, j-0.5, k} \\
& +[\mathbf{V} \cdot(S \mathbf{n})]_{i, j, k+0.5}-[\mathbf{V} \cdot(S \mathbf{n})]_{i, j, k-0.5}
\end{aligned}
$$

где $\Omega_{i, j, k}$ - объем ячейки, $q_{i, j, k}-$ среднее значение по ячейке и $S$ - площадь грани ячейки. Дробные индексы указывают, с какой стороны ячейки берется поток в квадратных скобках.

Разумеется, соотношение (2) является всего лишь формальной аппроксимационной схемой, для реализации которой следует построить интерполяционные формулы, по которым потоки в квадратных скобках интерполируются по значениям потоков в близлежащих центрах ячеек.

В пространственной аппроксимации потоков конвективные потоки на гранях ячеек интерполируются по данным в центрах ячеек посредством двух интерполяционных операторов: характеристического оператора первого порядка и ENO (Essentially non-Oscillatory Существенно неосцилляционного) оператора высокого порядка. Шаблон характеристической схемы первого порядка, фактически применяемой при релаксации, состоит из одной точки, выбранной по знаку соответствующего собственного числа.

Метод ENO (предложенный А. Хартеном и С. Ошером [8] и затем упрощенный С.-В. Шу и С. Ошером [9] применяется посредством выбора интерполяционного шаблона по локальным характеристикам и гладкости потоков, и может изменяться по ходу итераций. При этом применяется характеристическая декомпозиция, и интерполяция производится в соответствующих характеристических полях. Интерполяционный шаблон ENO (типично состоящий из трех точек в нашей имплементации) определяется отдельно в каждом характеристическом поле, сначала по знаку соответствующего собственного числа и затем в соответствии с гладкостью 
интерполируемых потоков. Чтобы вернуться к декартовым потокам после интерполяции, значения интерполированных характеристических потоков проектируются обратно.

Мы также используем метод поправки на дефект, в котором целевая дискретизация уравнений на самой тонкой сетке отличается от дискретизации, применяемой при многосеточной релаксации. Для релаксации используется аппроксимационный оператор первого порядка точности, а поправка на дефект вычисляется посредством аппроксимации ENO высокого порядка.

Таким образом, мы применяем схему ENO только при вычислении поправки на дефект, а большая часть работы осуществляется посредством относительно дешевой в вычислительном отношении характеристической схемы первого порядка.

Вязкие члены аппроксимируются напрямую. Для интегрирования по времени используется схема Рунге-Кутта третьего порядка, сохраняющая полную вариацию (TVD), разработанная С.-В. Шу и С. Ошером [9]. Более подробное описание численного метода дано в работах $[10,11]$.

В качестве поисковой машины оптимизация использует генетический алгоритм. Генетические алгоритмы (ГА), основанные на комбинации детерминистических и вероятностных подходов, имитируют эволюционный процесс в природе. В качестве базового варианта используется ГА с плавающей точкой и следующие генетические операторы: турнирная селекция, одноточечный оператор размножения, неравномерная мутация и элитизм.

В задаче оптимизации аэродинамических форм учет ограничений на решение исключительно важен. Причина этого в том, что (как и во многих реальных задачах) оптимальное решение не является локальным минимумом. Напротив, оптимум находится на пересечении гиперповерхностей различной размерности, и положение этих поверхностей заранее не известно.

Применяемый нами принципиально новый подход [1214] к учету нелинейных ограничений можно очертить следующим образом.

- Изменение традиционной стратегии, в которой маршрут поиска может проходить только через допустимые (удовлетворяющие ограничениям) точки, посредством допущения маршрутов, проходящих как через допустимые, так и через недопустимые точки. Основная идея этого подхода состоит в том, что информация из „запретных“ (т.е. не удовлетворяющих ограничениям) областей может оказаться очень важной, и путь к оптимальной точке, пролегающий через эти области, может оказаться существенно короче.

- С этой целью мы строим расширение целевой функции, производя ее оценку также и в недопустимых точках. Это оказалось возможным в силу одного из базовых свойств ГА: в противоположность классическим методам оптимизации ГА допускают негладкие расширения целевых функций.
Стандартные ГА обладают низкой вычислительной эффективностью в тех случаях, когда расчет целевой функции является вычислительноемким (как это имеет место в нашем случае при решении уравнений Навье-Стокса).

Чтобы эффективно имплементировать ГА, было необходимо значительно повысить вычислительную эффективность. Дело в том что традиционное использование ГА требует очень большого объема вычислений (даже алгоритм с населением $M=100$ требует - в случае 200 поколений - по меньшей мере 20000 решений полных уравнений Навье-Стокса), что практически является абсолютно неприемлемым.

Требования вычислительной эффективности диктуют следующие свойства алгоритма: число точных расчетов уравнений Навье-Стокса должно быть очень ограничено и тем не менее алгоритм должен обеспечить быстрый и достаточно точный расчет целевой функции при генетическом поиске.

Чтобы преодолеть это, мы используем метод аппроксимационных моделей в специально разработанной нами форме метода локальных аппроксимаций, в котором функционалы на решении (такие как подъемная сила и сопротивление) аппроксимируются на основе локальной базы данных. База данных строится посредством решения полных уравнений Навье-Стокса в дискретной окрестности текущей базовой точки в пространстве поиска.

Для обеспечения точности оптимального поиска мы сочетаем использование приближенных и „точных“ (полученных на основе полных уравнений Навье-Стокса) решений с помощью принципа „предикции-верификации“. На стадии предикции одновременно производится генетический поиск на ряде вложенных поисковых областей. Поиск на каждой такой области порождает (суб)оптимальную точку, и на стадии верификации весь набор таких точек-,,кандидатов“" проверяется посредством решения полных уравнений Навье-Стокса, тем самым определяя финальную оптимальную точку.

С целью преодоления локальной природы описанной выше аппроксимации и обеспечения глобальности поиска производятся внешние итерации, так что в каждой итерации финальный оптимум служит отправной точкой для следующей итерации.

Фактически мы используем гибридный ГА, сочетающий внешние итерации с общепринятым генетическим поиском в ограниченных областях пространства поиска. Количество внешних итераций, необходимых для сходимости всего процесса, обычно не превышает 8-10.

Чтобы сделать оптимизацию аэродинамических форм практически выполнимой, мы разработали высокомасштабируемую имплементацию на параллельных компьютерах. В этой имплементации мы используем пять переплетающихся уровней параллелизации.

На уровне 1 параллелизовано вычисление целевой функции (решение уравнений Навье-Стокса). В ней используется метод геометрической декомпозиции. Благодаря тому, что полная вычислительная область состав- 
лена из блоков, делается возможным распределить группы блоков по ведомым процессорам, контролируемым ведущим процессором. Каждый ведомый процессор выполняет вычисления в ячейках блоков, распределенных на него. На каждой итерации происходит обмен приграничной блочной информацией между процессорами, так что граничные полосы ячеек каждого блока получают данные из граничных полос соседних с ними блоков. Количество передаваемой информации пропорционально размеру сеточного шаблона (по большей части он равняется единице). Параллельная эффективность уровня 1 превышает 90\% ([15]).

Уровень 1 задействован совместно с уровнем 2, который осуществляет параллельное сканирование поискового пространства (обработка тестируемых геометрий). Уровни 1 и 2 улучшают вычислительную эффективность той части алгоритма, которая относится к расчету обтеканий, в то время как на уровнях 3-5 параллелизуется собственно оптимизационный поиск. На уровне 3 осуществляется параллелизация генетического поиска. Параллелизация на уровне 3 происходит во взаимодействии с уровнем 4, который осуществляет оптимальный поиск на ряде поисковых областей. Уровень 5 осуществляет параллельную генерацию сеток. Параллелизация была осуществлена на многопроцессорном кластере с помощью стандартного пакета PVM.

\section{Результаты численных решений}

Одним из важнейших вопросов, возникающих при использовании алгоритма автоматического оптимального аэродинамического проектирования для решения практических задач по проектированию летательного аппарата, является вопрос о том, насколько результат такого проектирования зависит от начальной формы оптимизируемой поверхности.

С практической точки зрения очень важно, чтобы алгоритм давал хорошие результаты не только в случае, когда начальная геометрия обладает приемлемыми аэродинамическими характеристиками, но и в случае, когда исходная форма в точках проектирования имеет высокий уровень полного сопротивления.

С математической точки зрения возникает вопрос об устойчивости алгоритма автоматического оптимального проектирования к начальной форме. Иными словами, если мы проведем две оптимизации с одинаковым условиями и ограничениями, но для двух сильно отличающихся друг от друга начальных геометрий, то насколько будут отличаться друг от друга две полученные оптимальные геометрии?

В связи с этим в настоящей работе были проведены исследования устойчивости использованного алгоритма автоматического оптимального проектирования к начальной форме изолированного трехмерного крыла широкофюзеляжного дальнемагистрального самолета.
При проведении оптимального аэродинамического проектирования оптимизируемое крыло сохраняло постоянную форму в плане и было представлено пятью секциями по размаху крыла: бортовая секция $Z=3.04 \mathrm{~m}$; 1-я промежуточная секция $-Z=9.0 \mathrm{~m}$; 2-я промежуточная секция $-Z=16.5 \mathrm{~m}$; 3-я промежуточная секция $-Z=22.5 \mathrm{~m}$; концевая секция $Z=30.0 \mathrm{~m}$ (где $Z-$ координата вдоль размаха крыла).

Крыло имеет постоянный угол скоса передней кромки, равный $33.5^{\circ}$, и следующие углы скоса задней кромки: $4.82^{\circ}$ - между бортовой и 1-й промежуточной секцией; $16.58^{\circ}$ - между 1-й и 2-й промежуточными секциями; $22.86^{\circ}$ - между 2-й и 3-й промежуточными секциями; $24.26^{\circ}$ - между 3-й промежуточной и концевой секциями.

Были наложены следующие габаритные ограничения на относительные толщины оптимизируемых профилей: относительная толщина бортового профиля была равна $13.25 \%$, относительная толщина профиля в 1-й промежуточной секции составляла $11.1 \%$, относительная толщина профиля во 2-й промежуточной секции - 9.2\%, в 3-й промежуточной секции - $8.7 \%$, а относительная толщина концевого профиля крыла равнялась $8 \%$.

Для гарантированного размещения топливного бака в крыле самолета дополнительно были выставлены следующие ограничения на относительную толщину секций крыла в заданных значениях продольной координаты $X / C(C-$ величина локальной хорды секции крыла):

- бортовая секция $-11.8 \%$ (при $X / C=0.16)$ и $8.9 \%$ (при $X / C=0.65)$;

- 1-я промежуточная секция - 8.9\% (при $X / C=0.16)$ и $7.7 \%($ при $X / C=0.65)$;

- 2-я промежуточная секция - 7.5\% (при $X / C=0.16)$ и $6.4 \%$ (при $X / C=0.65)$;

- 3-я промежуточная секция - 6.9\% (при $X / C=0.16)$ и $6.4 \%$ (при $X / C=0.65)$;

- концевая секция - $6.5 \%$ (при $X / C=0.16$ ) и $5.9 \%$ (при $X / C=0.65$ ).

Были рассмотрены два варианта задания начальных форм крыла.

В первом варианте секционные профили совпадали c исходной геометрией широкофюзеляжного дальнемагистрального самолета. Все эти профили имели суперкритический характер, что и требуется для полета на высоких трансзвуковых числах Маха.

Во втором варианте начальная геометрия была намеренно выбрана абсолютно непригодной с аэродинамической точки зрения. В частности, вместо суперкритического профиля на 1-й промежуточной секции крыла был поставлен симметричный профиль, в котором верхняя поверхность совпадала с верхней поверхностью исходного суперкритического профиля. Дополнительно полученный симметричный профиль был отскалирован, чтобы он отвечал всем требуемым ограничениям: имел максимальную относительную толщину $11.1 \%$ и относительную толщину $8.9 \%$ (при $X / C=0.16$ ) и $7.7 \%$ (при $X / C=0.65)$. 


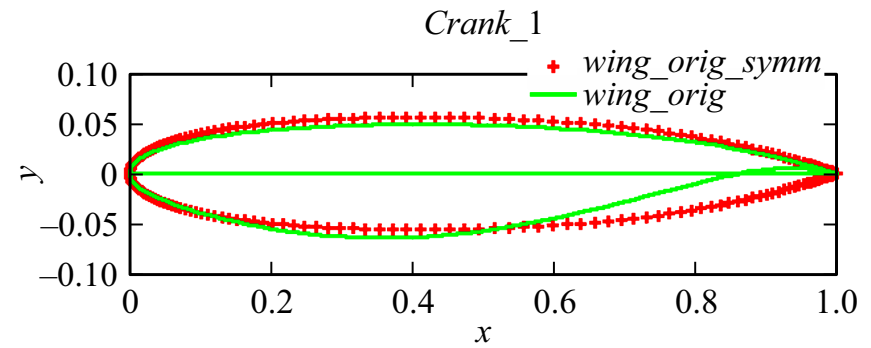

Рис. 1. Сравнения начальных профилей в 1-ой промежуточной секции изолированного трехмерного крыла широкофюзеляжного дальнемагистрального самолета.

Сравнение данных начальных профилей для двух вариантов оптимизации представлено на рис. 1 .

Для проведения оптимизации использовался программный продукт OPTIMENGA_AERO, ранее разработанный авторами и предназначенный для автоматического оптимального аэродинамического проектирования трехмерных изолированных крыльев произвольной в плане формы с учетом конструктивных параметров и конструктивных ограничений.

В качестве точки аэродинамического проектирования была выбрана точка на крейсерском режиме полета: $M=0.86, C_{y}=0.5$.

Дополнительным аэродинамическим ограничением являлось ограничение на момент тангажа, необходимое для обеспечения устойчивости полета. При проведении оптимального аэродинамического проектирования требовалось, чтобы момент тангажа всей конфигурации был не меньше, чем момент тангажа 1-й начальной конфигурации.

Расчетная сетка для пятисекционного крыла состояла из 4 блоков: двух блоков, прилегающих к крылу, и двух блоков на следе крыла. Благодаря использованию условия симметрии на центральной линии крыла сетка строится только для половины крыла (от плоскости симметрии до численной „бесконечности“).

Блоки около крыла имеют топологию $\mathrm{C}-\mathrm{O}(\mathrm{C}-$ по маршевой координате и $O-$ по размаху крыла). Каждый из этих двух блоков содержит 64 расчетные ячейки по маршевой координате, 32 расчетные ячейки в направлении, нормальном к поверхности крыла, и 52 расчетные ячеек в направлении по размаху крыла, в общей сложности $64 \times 32 \times 52=106496$ расчетных ячеек в блоке.

Блоки на следе имеют топологию $H-O$ ( $H-$ по маршевой координате и $O-$ по размаху крыла). Каждый из этих двух блоков содержит 32 расчетные ячейки по маршевой координате, 32 расчетные ячейки в направлении, нормальном к поверхности крыла, и 52 расчетные ячейки в направлении по размаху крыла, итого $32 \times 32 \times 52=53248$ расчетных ячеек в блоке. Таким образом, тонкая сетка вокруг половины крыла содержит 319488 расчетные ячейки. Для повышения точности расчетов шаги сетки были неравномерными, и сетка сгущалась в области течения с большими градиентами. В частности, шаг сетки вдоль поверхности крыла в окрестности передней кромки составлял порядка $0.2 \%$ от локальной хорды крыла, а в окрестности задней кромки был порядка $0.45 \%$ от локальной хорды крыла. Величина 1-го шага по направлению по нормали к поверхности крыла составлял $0.02 \mathrm{~mm}$.

Анализ распределения давления на верхней поверхности крыла в точке проектирования при $M=0.86$, $C_{y}=0.50$, подтвердил предположение, что симметричный профиль в середине крыла абсолютно непригоден для данных условий обтекания. Об этом же говорит и сравнение поляр сопротивления для двух вариантов задания начальной формы крыла при $M=0.85$, приведенной на рис. 2. Все это свидетельствует о том, что с аэродинамической и геометрической точек зрения 2-й вариант начальной геометрии крыла находится очень далеко от оптимального решения.

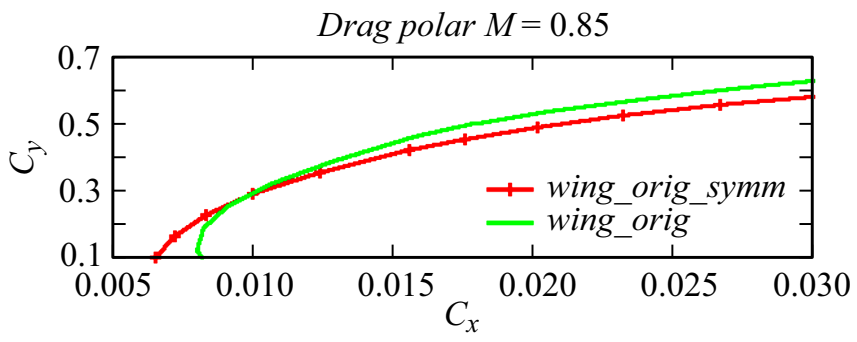

Рис. 2. Сравнения поляр сопротивления для двух вариантов начальной формы изолированного трехмерного крыла широкофюзеляжного дальнемагистрального самолета.

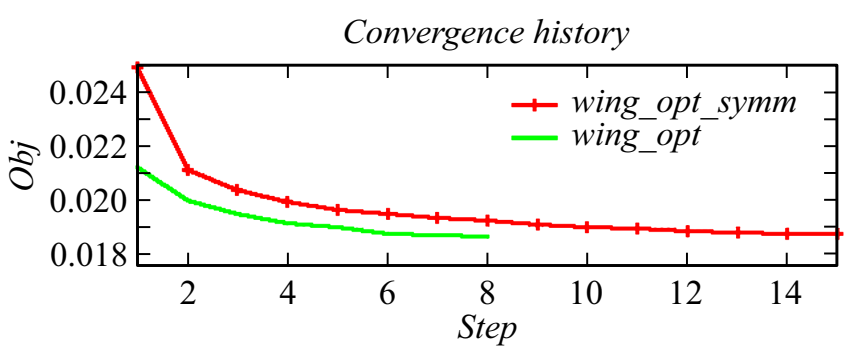

Рис. 3. Сходимость оптимизационного процесса.

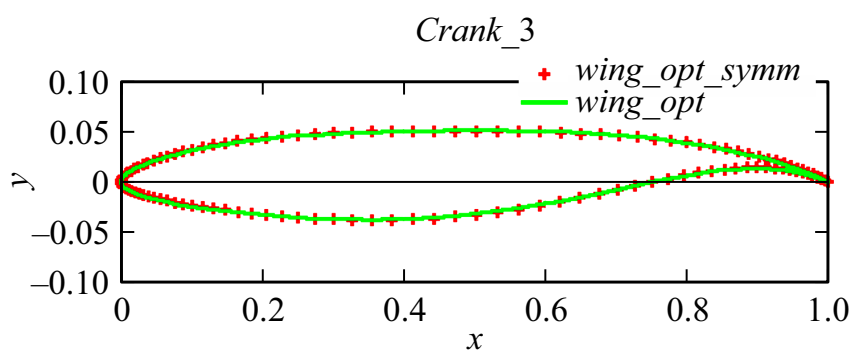

Рис. 4. Сравнения оптимальных профилей в 3-ей промежуточной секции изолированного трехмерного крыла широкофюзеляжного дальнемагистрального самолета для двух вариантов оптимизации. 
Перейдем теперь к сравнениям результатов этих двух одноточечных оптимизаций. Для получения оптимального решения для 1-го варианта задания начальной формы потребовалось 8 шагов оптимизационного алгоритма, в то время как для достижения сходимости для 2-го варианта было необходимо 15 шагов. Соответствующая кар-

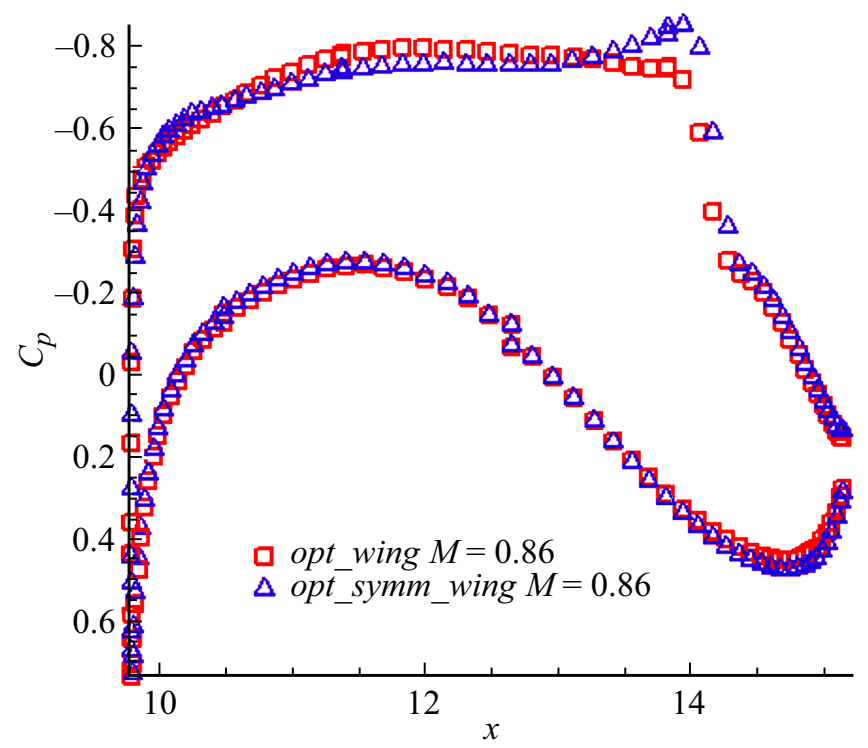

Рис. 5. Сравнение распределений давления в сечении $Z=13.46$ m по размаху крыла при $C_{y}=0.50$ для $M=0.86$ для двух вариантов оптимизации.

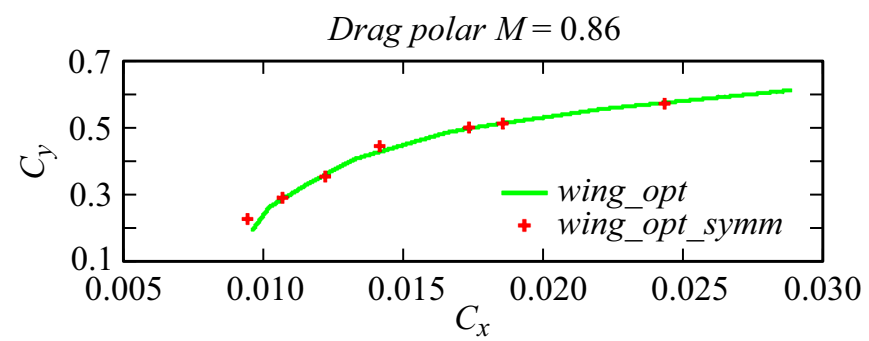

Рис. 6. Сравнения поляр сопротивления оптимального изолированного трехмерного крыла широкофюзеляжного дальнемагистрального самолета для двух вариантов оптимизации при $M=0.86$

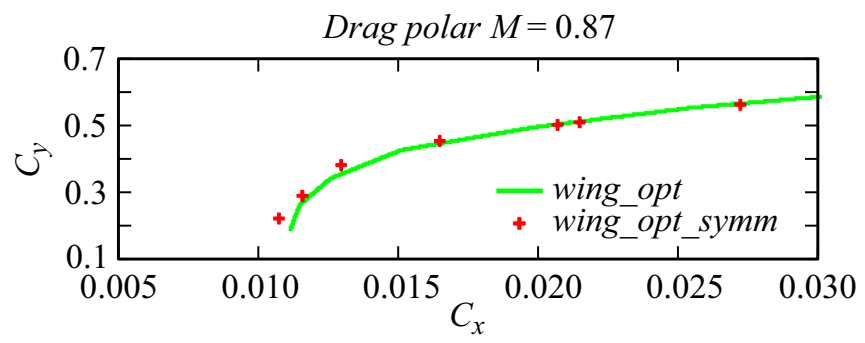

Рис. 7. Сравнения поляр сопротивления оптимального изолированного трехмерного крыла широкофюзеляжного дальнемагистрального самолета для двух вариантов оптимизации при $M=0.87$.

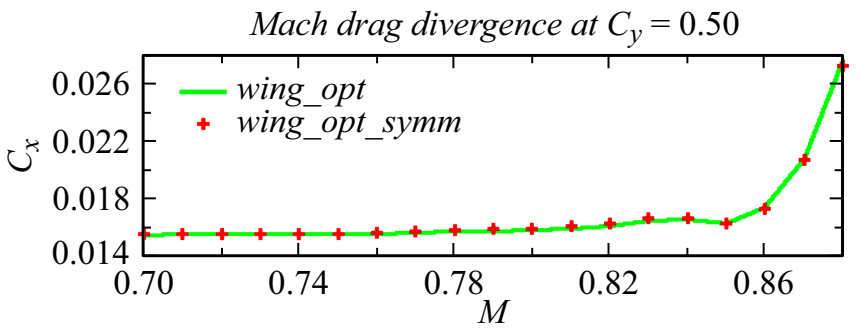

Рис. 8. Сравнения зависимость сопротивления от числа Маха при фиксированном $C_{y}=0.5$ оптимального изолированного трехмерного крыла широкофюзеляжного дальнемагистрального самолета для двух вариантов оптимизации при $M=0.87$.

тина сходимости оптимизационного процесса приведена на рис. 3.

Результаты оптимизации оказались очень близкими, и с геометрической точки зрения оптимальные геометрии крыла были практически идентичными. Соответствующий пример сравнения секционных сечений двух оптимальных крыльев при $Z=13.46$ m приведен на рис. 4 .

$\mathrm{C}$ аэродинамической точки зрения оптимальные крылья также оказались очень близки друг к другу. В частности, в основной точке проектирования $M=0.86$, $C_{y}=0.50$ сопротивление оптимального крыла для 1-го варианта составило 174.3 аэродинамических каунта против 173.6 для 2-го варианта.

Соответствующее распределение давления на поверхности оптимального крыла и секционные распределения давления оказались очень близки. Пример такого сравнения для секционного сечения $Z=13.46$ m приведен на рис. 5.

Полученные оптимальные решения оказались аэродинамически близки не только локально в точке проектирования. Малые отличия двух оптимальных крыльев сохранялись и в широком диапазоне условий полета в окрестности точки проектирования как по числам Маха, так и по углу атаки. Соответствующие сравнения интегральных характеристик оптимальных геометрий полученных для двух вариантов задания начальной формы крыла приведены на рис. 6-8. На рис. 6, 7 приведены поляры сопротивления при $M=0.86$ и $M=0.87$, а на рис. 8 - зависимости сопротивления от числа Маха при фиксированном $C_{y}=0.5$.

\section{Заключение}

Анализ полученных аэродинамических характеристик оптимальных крыльев широкофюзеляжного дальнемагистрального самолета для двух сильно отличающихся вариантов задания начальной формы крыла, показывает, что используемый алгоритм оптимального аэродинамического проектирования является устойчивым к заданию начальной формы, поскольку:

- оптимальные крылья обладают практически одним и тем же малым волновым сопротивлением в основной 
точке проектирования $C_{y}=0.50, M=0.86\left(C_{X}=173.6\right.$ каунта и $C_{X}=174.3$ каунта);

- формы оптимальных крыльев очень блики друг к другу;

- оптимальные крылья обладают очень близкими (практически идентичными) интегральными аэродинамическими характеристиками в широком диапазоне изменения условий полета.

Работа выполнена при финансовой поддержке Минобрнауки РФ в рамках реализации Проекта RFMEFI57617X0103.

\section{Список литературы}

[1] Jameson A., Martinelli L., Vassberg J. // Proc. of the $23^{\text {rd }}$ International Congress of Aeronautical Sciences. Toronto, Canada, 2002. 25 p.

[2] Vassberg J., Jameson A. Aerodynamic Shape Optimization Part 1: Theoretical Background. Brussels: Von Karman Institute, $2006.30 \mathrm{p}$.

[3] Alauzet F., Mohammadi B., Pironneau O. Variational Analysis and Aerospace Engineering: Mathematical Challenges for Aerospace Design. Springer: Optimization and Its Applications, 2012. Vol. 66. 18 p.

[4] Mohammadi B., Pironneau O. // Annu. Rev. Fluid Mech. 2004. Vol. 36. P. $11.1-11.25$.

[5] Gauger N. // ECMI Newsletter. 2007. N 41.4 p.

[6] Kroll N., Gauger N., Brezillon J., Dwight R. et al. // J. Comput. Appl. Math. 2007. Vol. 203. N 2. P. 397-411.

[7] Epstein B., Jameson J., Peigin S., Roman D., Harrison N., Vassberg J. // J. Aircraft. 2009. Vol. 46. N 2. P. 526-541.

[8] Harten A., Osher S. // I.SIAM J. Numer. Analys. 1987. Vol. 24. P. 279.

[9] Shu C.-W., Osher S. // J. Comput. Phys. 1988. Vol. 77. P. 439-471.

[10] Epstein B., Averbuch A., Yavneh I. // J. Comput. Phys. 2001. Vol. 168. P. 316-338.

[11] Epstein B., Peigin S.V. // Int. J. Comput. Fluid. D. 2004. Vol. 18. N 3. P. 289-293.

[12] Peigin S., Epstein B. // Int. J. Numer. Meth. Fl. 2004. Vol. 45. P. $1339-1362$.

[13] Epstein B., Peigin S. // AIAA J. 2005. Vol. 43. N 9. P. 1946-1957.

[14] Peigin S., Epstein B. // Aerosp. Sci. Technol. 2008. Vol. 12. N 2. P. 125-134.

[15] Peigin S., Epstein B. // J. Supercomput. 2004. Vol. 29. P. $243-263$. 